\title{
TRATAMIENTO COGNITIVO-CONDUCTUAL DE LA COMPRA COMPULSIVA. UN CASO CLÍNICO
}

\author{
Mónica Gómez Peña \\ Biblana Sans \\ Eva María Álvarez-Moya \\ María Neus Aymaml \\ Susana Jiménez Murcla
}

Hospital Universitario de Bellvitge. Unidad de Juego Patológico. Servicio de Psiquiatría.

\section{RESUMEN}

Algunas personas manifiestan frecuentemente intensos deseos por comprar objetos innecesarios, presentando dificultades para controlar la conducta de compra. Tras los episodios de compra compulsiva suelen manifestar sentimientos de culpabilidad, los objetos comprados no se suelen utilizar, o se almacenan y, a largo plazo, esta conducta les aporta consecuencias negativas en el ámbito personal, familiar, laboral y económico.

A pesar de que la compra compulsiva no ha sido incluida en las clasificaciones diagnósticas oficiales (DSM-IV-TR o CIE-10), algunos autores sugieren que podría considerarse como un trastorno del control de los impulsos no clasificados en otros apartados, mientras que otros observan más similitudes con los criterios de abuso o dependencia de sustancias, los trastomos obsesivo-compulsivos o los trastornos afectivos. Los estudios realizados indican que es un trastomo

Correspondencia: Mónica Gómez Peña. Psicólogo Adjunto. Unidad de Juego Patológico. Servicio de Psiquiatría. Hospital Universitario de Bellvitge. C/ Feixa Larga, s/n. 08907 Hospitalet de Llobregat. Barcelona. España. Tel.:93 26079 88. e-mail: monicagomez @csub.scs.es. 
con una prevalencia del $1.1 \%$ en la población general, que afecta principalmente a mujeres. Diferentes estudios coinciden en la necesidad de abordar este trastorno mediante tratamiento psicológico.

Presentamos un estudio de caso en el que hemos aplicado, con éxito, un tratamiento cognitivo-conductual a una mujer de 59 años con compra compulsiva, considerando la compra compulsiva como una adicción psicológica no ligada al uso de sustancias. Las intervenciones terapéuticas habitualmente empleadas en el tratamiento psicológico de las adicciones químicas y del juego patológico, como el control de estímulos, prevención de recaídas, exposición con prevención de respuesta y terapia de pareja, son aplicadas y discutidas. Mostramos, asimismo, los resultados del seguimiento al mes y a los 3 meses.

Palabras clave: COMPRA COMPULSIVA, TRASTORNOS DEL CONTROL DE LOS IMPULSOS, ADICCIÓN COMPORTAMENTAL, TRATAMIENTO COGNITVO-CONDUCTUAL.

\section{SUMMARY}

Some people frequently manifest an intense desire to purchase unnecessary items and have difficulty in controlling this shopping behaviour. After episodes of compulsive shopping, they usually express feelings of guilt, the objects purchased are not used or are stored away and in the long term, this behaviour has negative consequences on a personal, familial, occupacional and financial level. In spite of the fact that compulsive shopping has not been included in official, diagnostic classifications (DSMIV-R or CIE-10), some authors suggest that it could be considered an impulse control disorder not classified elsewhere. On the other hand, others observe more similarities with substance abuse or dependence critenia, obsessive-compulsive disorders or affective disorders. The studies carried out so far report a prevalence of $1.1 \%$ for the disorder in the general population, and that it mainly affects women. Various reports 
agree on the need to deal with this disorder by means of psychological treatment.

We present a case study in which we have successfully applied a cognitive-behavioural approach to a 59 year old woman suffering from compulsive shopping. Compulsive buying was considered a psychological addiction not linked to substance abuse. Therapy habitually employed in the psychological treatment of substance addictions and pathological gambling, such as stimulus control, prevention of relapses, exposure with response prevention and couple therapy have been applied and discussed. Furthermore, we present the results of follow-up at one and three months.

Key words: COMPULSIVE BUYING, IMPULSIVE-CONTROL DISORDER, BEHAVORAL DEPENDENCE, COGNITVE-BEHAVIORAL TREATMENT.

\section{INTRODUCCIÓN}

El fenómeno de la compra compulsiva ya fue descrito por Kraepelin en 1915, denominándolo «oniomanía» o «mania de las compras» y considerándolo como un impulso patológico que afectaba principalmente a mujeres (cfr. Bleuler, 1924).

Durante los años 80 , desde una perspectiva que contemplaba el consumo como un fenómeno sociocultural, diversos autores realizaron investigaciones sobre los hábitos de consumo con el objetivo de determinar el perfil del abuen consumidor». No obstante, los resultados de estas investigaciones indicaron que muchas personas presentaban hábitos de consumo inadecuados (Faber, O'Guinn y Krych, 1987). En un trabajo posterior estos autores definieron la compra incontrolada como «una forma inapropiada de conducta de compra, excesiva, que altera la existencia del individuo, el cual presenta una propensión al consumo impulsivo» (O'Guinn y Faber, 1989) y, más adelante, como acompras repetitivas y crónicas que se convierten en la respuesta primaria a sucesos o eventos negativos. Resultan difíciles de parar y conllevan consecuencias dañinas» (Faber y O'Guinn, 1992). 
A pesar de que existen escasos estudios epidemiológicos, diferentes autores afirman que la tasa de prevalencia en la población general de este trastorno se sitúa en un 1.1\% (Faber y O'Guinn, 1992; Lejoyeux, Adès, Tassain y Solomon, 1996). Diversos estudios han arrojado como resultado que es un trastorno que afecta principalmente a mujeres: el $92 \%$ de la muestra evaluada en el estudio de Christenson y cols. (1994), y el 80\% de la muestra en el estudio de McElroy, Keck, Pope, Smith, y Strakowski (1994) y Schlosser, Black, Repertinger y Freet (1994). Es un trastorno crónico, con pocos períodos de remisión, aunque para algunas personas puede ser episódico (Christenson y cols., 1994; Schlosser y cols., 1994). Su inicio se sitúa a principios de la vida adulta, entre los 18 y los 30 años (Christenson y cols., 1994; Schlosser $y$ cols., 1994; McElroy y cols., 1994). Desde un punto de vista conductual, las personas que presentan compra compulsiva prefieren comprar solas y comprar artículos para ellas mismas (Schlosser $y$ cols., 1994). Principalmente, los objetos comprados son ropa, zapatos, joyas y artículos de perfumería, en el caso de las mujeres, y artículos de electrónica, vídeos, equipos de música y accesorios para el coche, en el caso de los hombres. Generalmente, los artículos comprados se devuelven, no se utilizan o se almacenan (Christenson y cols., 1994; Schlosser y cols., 1994). Estas personas tienden a un mayor uso de las tarjetas de crédito, 8 de cada 10 poseen una tarjeta al descubierto y un 7\% tienen más de 10 tajjetas (Adès y Lejoyeux, 2003). Es un trastomo que se ha asociado a comorbilidad psiquiátrica, principalmente con trastornos de ansiedad $(41 \%)$, trastornos por consumo de sustancias (30\%), trastornos afectivos (28\%) y trastornos de la conducta alimentaria (17\%) (Schlosser y cols., 1994). Otros estudios realizados arrojan datos similares (Christenson y cols., 1994). Siguiendo esta línea, Black, Repertinger, Gaffney y Gabel (1998) hallaron la presencia comórbida con depresión mayor $(60.6 \%)$, asi como la existencia de trastornos psiquiátricos en tamiliares de primer grado, principalmente alcoholismo (19.7\%), depresión (18.3\%) y trastomos por consumo de sustancias $(5.1 \%)$.

No existe una definición operacional de la compra compulsiva en la nomenclatura psiquiátrica oficial. Es por ello que aparecen conclusiones divergentes en tomo a la clasificación de este trastorno. Según los estudios realizados, se ha considerado la compra compulsiva 
como un trastomo del control de los impulsos no clasificados en otros apartados (Dittmar y Drury, 2000; Ninan, y cols., 2000). No obstante, otros estudios apuntan que comparte características similares a los trastornos obsesivos compulsivos (McElroy y cols., 1994; Christenson $y$ cols., 1994; Black, Monahan, Schlosser, y Repertinger, 2001) o que se asocia a los trastomos del estado de ánimo (McElroy, Satlin, Pope, Keck y Hudson, 1991). Por otra parte, otros autores consideran la compra compulsiva como una adicción conductual (Echeburúa, 1999; Elliott, 1994; Glatt y Cook, 1987; Krych, 1989; Marks, 1990).

Del mismo modo, no existen datos consistentes en cuanto al tratamiento de elección de este trastomo. No obstante, la mayoría de las investigaciones centradas en el tratamiento de la compra compulsiva coinciden en la necesidad de un tratamiento psicológico. Se ha considerado el uso de terapia farmacológica, terapia psicológica o la combinación de ambas. Algunos autores proponen un abordaje psicofarmacológico centrado en el uso de antidepresivos (McElroy y cols.,1991; Black, Monahan y Gabel, 1997; Aboujaoude, Gamel y Koran, 2003). McElroy y cols., (1994) aconsejan tratamiento farmacológico combinado con psicoterapia de apoyo u orientada al insight. Los estudios llevados a cabo desde una perspectiva psicodinámica ofrecen resultados poco claros (Krueger, 1988; Lawrence, 1990; Winestine, 1990). En la revisión realizada por Black (1996), este autor sugiere que estas personas se beneficiarían de terapia conductual basada en exposición con prevención de respuesta, terapia cognitivo-conductual y tratamiento grupal. Siguiendo esta línea, Adés y Lejoyeux (2003), Echeburúa (1999) y Marks (1990) proponen tratamiento cognitivo-conductual para el abordaje de este trastorno. Por otro lado, Miltenberger y cols., 2003) sugieren que estas personas se beneficiarian del aprendizaje de respuestas de afrontamiento adecuadas frente a emociones negativas.

El objetivo de este trabajo es presentar un caso clínico de compra compulsiva, tratado desde una orientación cognitivo-conductual, con el propósito de describir las técnicas psicológicas aplicadas y de analizar los resultados obtenidos. Se ofrecen, además, los datos del seguimiento al mes y a los 3 meses. 


\section{DESCRIPCIÓN DEL CASO}

\section{Datos soclodemográficos}

M. es una mujer de 59 años de edad, natural de Barcelona y con estudios secundarios. Trabaja desde hace 27 años como monitora escolar, con contrato laboral discontinuo. Está casada y tiene una hija de 32 años y un hijo de 27 años. En la actualidad, su hija está casada y la paciente vive con su marido y con su hijo.

\section{Problemas que presenta}

M. presentaba un problema de gastos excesivos de varios años de evolución. Experimentaba frecuentemente una necesidad de comprar que le llevaba a realizar numerosos episodios, en los que cornpraba de forma no planificada diversos objetos, sin considerar su precio, sus posibilidades económicas o la utilidad de los mismos. Desde hace unos 7 años este comportamiento se convierte en un problema. Los episodios de compra progresivamente se hicieron más frecuentes, siendo sus desencadenantes principales estímulos extemos y estados emocionales negativos. Durante los episodios experimentaba sensación de pérdida de control sobre la conducta. El esconder los objetos comprados, los pequeños hurtos de dinero a famlliares, los múltiples créditos bancarios, las mentiras y la sensación de culpabilidad eran frecuentes. Su marido descubrió que habla falsificado su firma para conseguir un préstamo de una financiera, cuando comenzaron a llegar los recibos del préstamo a su casa. M. habla ocultado a todos los miembros de su familia su problema con las compras durante muchos años. El marido y los hijos se sentían muy desbordados emocionalmente por haber descubierto el problema de M. y por las consecuencias económicas que este problema ocasionaba. La relación conyugal se habla deteriorado notablemente y su marido consideraba la posibilidad de divorciarse. La deuda económica contraída ascendía a 15.000 . Por todo ello, la paciente mostraba bajo estado de ánimo, ansiedad y marcados sentimientos de culpabilidad. 


\section{Análisis funcional de la conducta de compra compulsiva}

\section{Antecedentes}

\section{Desencadenantes externos.}

-Disponer de dinero en efectivo.

-Estar en grandes almacenes, hipermercados, pequeños comercios, tiendas "todo a 100" y mercadillos.

-Disponer de tiempo libre, especialmente por las tardes, al terminar su horario laboral.

- Ver un escaparate.

-Anuncios comerciales de un nuevo producto.

-Periodos de rebajas.

- Observar gran variedad de marcas de un mismo producto.

-Discusiones con su marido.

-Discusiones con sus hijos.

Desencadenantes internos:

-Aumento de peso.

-Tristeza.

-Sentimientos de baja autoestima.

-Sentimientos de frustración.

-Sentirse "anulada" en casa.

\section{Respuestas}

\section{Motoras}

-Mostraba urgencia para iniciar la conducta de compra y, una vez iniciada, experimentaba dificultades para pararla.

-Ocurrencia de varios episodios de compra compulsiva a la semana.

-Compraba sola.

-El dinero lo obtenía de su nómina y de solicitar créditos a bancos y financieras.

-Nunca usaba tarjetas de crédito al efectuar sus compras, sino que compraba siempre utilizando dinero en efectivo.

-El gasto medio por episodio de compra era de unos $50 €$ y la cantidad máxima gastada fue de unos $200 €$. 
-Se sentla irritable e inquieta en el caso de manifestar deseo de compra y no disponer de dinero.

-No comparaba precios y compraba cosas caras.

-En caso de haber distintas marcas, compraba varias y en gran cantidad, aún siendo consciente de que no iba a usar esos productos.

-Compraba principalmente varios regalos para sus familiares, ropa para sus hijos o para ella, comida, articulos para la casa, como múltiples utensilios de cocina y artículos de decoración, libros para su hijo, cosméticos (M. refería que habitualmente no se maquillaba), productos de adelgazamiento, coleccionables. Además, utilizaba el taxi para efectuar sus compras, cuando habitualmente usaba el transporte público.

Fisiológicas

-Tensión muscular, taquicardia, hiperactivación autonómica.

\section{Cognitivas}

-Cogniciones centradas en comprar regalos para agradar a los demás.

-Expectativas de incrementar su autoestima ( «si llevo dinero me tratan mejor, me siento importantex).

\section{Emocionales}

-Euforia, sentimientos de seguridad, relajación.

\section{Consecuenclas}

\section{A corto plazo}

-Tras los episodios de compra M. se sentía relajada de forma inmediata, pero posteriormente experimentaba intensos sentimientos de culpabilidad.

-Excusas y mentiras para ocultar los gastos realizados y los objetos comprados.

- Tiraba muchos artículos comprados (comida) o no los usaba (ropa, maquillaje).

-Escondía y almacenaba regalos que había comprado con antelación.

-Satisfacción al regalar varios objetos a la vez.

-Preocupaciones constantes por obtener dinero.

-En caso de haber agotado el dinero que disponible, manifestaba malestar subjetivo que se aliviaba solicitando otro crédito. 
-Actitud de hipervigilancia hacia llamadas de teléfono o correo que llegaban a su casa para poder ocultar a su familia los créditos que había solicitado a las financieras.

-Insomnio de mantenimiento, despertares frecuentes, sensación de sueño no reparador.

-Ansiedad psíquica.

- Irritabilidad.

-Discusiones frecuentes con sus familiares.

-Inquietud psicomotora, alteraciones gastroinstentinales.

-Tristeza, sentimientos de soledad.

A largo plazo

-Falsificación de la firma de su marido, con el objetivo de obtener un préstamo económico.

-En el ámbito personal: tristeza y ansiedad, astenia, anergia, sentimientos de culpa e insomnio de mantenimiento.

-En el ámbito familiar: marcado deterioro de la relación familiar, especialmente la conyugal.

-En el ámbito económico: la deuda contraída ascendía a una cantidad de $15.000 €$ ( 2 créditos a financieras y 1 préstamo bancario).

\section{Historia del problema}

M. describió su infancia caracterizada principalmente por un entorno familiar con importantes problemas económicos. Era la menor de 3 hermanas y comentaba que pasaba mucho tiempo junto a su padre. Este mostraba tendencia a vivir por encima de sus posibilidades económicas y a no gestionar adecuadamente el dinero. Frecuentemente compraba cuadros, ropa cara, hacía reformar repetidamente su casa, tenía un chofer para ir a su casa de verano (en lugar de obtener el carnet de conducir),... Como consecuencia de estos gastos, el padre de M. perdió su imprenta, empeñó joyas de su madre, fue objeto de embargos y llegó a timar, mentir e, incluso, pedir dinero a sus hijas. De niña, M. comenzó a coger a escondidas dinero a su madre para comprar golosinas, muñecas recortables,... Su madre utilizaba la negación como defensa, es decir, a pesar de los gastos excesivos que realizaba su marido y a pesar de que veía que $M$. compraba cosas desconociendo de dónde podría haber conseguido el dinero, no 
comentaba nada. La paciente en su juventud continuaba mostrando una marcada tendencia a gestionar inadecuadamente el dinero.

Tras su matrimonio, la paciente comienza a obtener dinero de las libretas y tarjetas de su marido, a escondidas, para poder realizar compras abundantes. Describía a su marido como autoritario, rígido, agresivo, con cambios de humor frecuentes, tacaño y con tendencia a gestionar y controlar excesivamente la economía familiar. Tenía costumbre de llevar una contabilidad exhaustiva de todos los gastos realizados por la familia para poder conocer detalladamente a fin de mes lo invertido en comida, perfumería, facturas, etc.

Hacía 8 años, M. realizó una consulta profesional, por iniciativa de su marido, debido a las consecuencias económicas que le habían aportado sus frecuentes episodios de compra. En 2 años había cogido a su marido $18.000 €$. La paciente admitía que gastaba excesivamente pero no precisó en qué lo gastaba. Como desencadenantes de su conducta de compra, la paciente describió bajo estado de ánimo reactivo a que su marido había iniciado una relación extramatrimonial hacia un año, constituyendo este hecho una condición que intensificó su problema de compra. En esta primera consulta se estableció la orientación diagnóstica de compra compulsiva y la paciente asistió a sesiones terapéuticas centradas en la realización de actividades alternativas.

Sin embargo, a lo largo de los siguientes años, la paciente continuó efectuando frecuentes episodios de compra, cada vez de mayor intensidad y, al mismo tiempo, su marido continuó manteniendo dicha relación extramatrimonial durante 7 años más. El problema con las compras fue acentuándose, así como el distanciamiento emocional entre ambos cónyuges.

Dos años y medio antes de consultar en nuestra unidad, apareció en la biografía de la paciente otro suceso vital significativo que influyó agravando su problema. M. fue intervenida quirúrgicamente, requiriendo una mastectomia y linfademectomía derecha con posterior tratamiento con radioterapia y quimioterapia. Comentó que acudió a una visita de control rutinaria y le indicaron la conveniencia de realizar la intervención quirúrgica en pocos dias, tras haberle diagnosticado una neoplasia mamaria. Su marido estaba de viaje, por motivos laborales y M. prefirió no comentarle nada. Tras la intervención quirúrgica, la paciente no percibió apoyo emocional por parte de su marido, pues se mostraba 
reservado y frío. Paralelamente, la paciente no aceptó su imagen corporal contribuyendo todo ello a que se deterioraran sus relaciones sexuales. De hecho, M. comentó que, desde entonces, no habían mantenido ningún contacto sexual y que, incluso, llevaban tiempo durmiendo en camas separadas. Los sentimientos de tristeza y soledad, junto con una valoración poco satisfactonía de su matrimonio, se asociaron a una progresión en la frecuencia de sus episodios de compra, así como a una preocupación constante por obtener dinero, llegando incluso a solicitar numerosos créditos a entidades bancarias y a financieras con el fin de continuar realizando episodios de compra. Las deudas económicas contraídas llevaron a la paciente a falsificar la firma de su marido con la intención de obtener un nuevo préstamo con el que solventarlas. Su marido lo descubrió indirectamente y ambos decidieron solicitar ayuda profesional.

\section{EVALUACIÓN PSICOLÓGICA Y RESULTADOS}

Tras la primera entrevista en nuestra unidad se inició un protocolo de evaluación psicológica consistente en la administración de los siguientes cuestionarios:

MMPI-2. (Inventario Multifésico de Personalidad de Minesota-2). (Minnesota Multiphasic Personality Inventory-2; Hathaway y McKinley, 1989).

Consta de 567 ítems a los que el sujeto debe responder "verdadero" - "falso". Proporciona puntuaciones en 10 escalas clínicas básicas, en escalas de contenido y en escalas suplementarias y evalúa características de la personalidad, tanto normal como patológica.

Los resultados obtenidos evidenciaron que la paciente presentaba un perfil significativamente elevado en las escalas clínicas Esquizofrenia, Depresión, Desviación Psicopática y Psicastenia. También destacaban puntuaciones significativamente elevadas en las escalas de contenido Depresión, Preocupación por la salud y Ansiedad.

MCMI-II. (Inventario Clínico Multiaxial de Millon-II). (Millon Clinical Mental Inventory-Il; Millon, 1990).

Consta de 175 ftems de contestación "verdad" o "falso" y ofrece puntuaciones en escalas de personalidad, escalas de personalidad patológica grave y escalas de síndromes clínicos. 
Los resultados obtenidos fueron significativamente elevados en las escalas Pasivo-Agresiva, Narcisista, Autodestructiva y Esquizoide.

$\mathrm{I}_{7}$.(Escala de Impulsividad $\mathrm{I}_{7}$ de Eysenck). (Eysenck's Impulsiveness Scale /; Eysenck, Pearson, Easting y Alsoop, 1985; Eysenck y Eysenck, 1991).

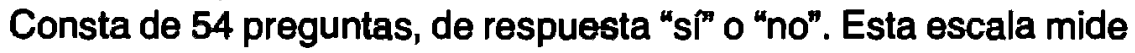
las vaniables Impulsividad, Aventura y Empatía. La puntuación obtenida es el sumatorio de los ítems recogidos para cada uno de los factores.

La paciente obtuvo una puntuación en el factor Impulsividad de 5 sobre 19; en Aventura, 1 de 16; y, en Empatía, 14 de un máximo de 18.

TCl-A (Inventario de Temperamento y Carácter, Revisado). (Temperament and Character Inventory Revised, Cloninger, 1999). Baremación española: Gutiérrez-Zotes y cols., 2004.

Consta de 240 ítems en los que el sujeto ha de describir cómo se siente o actúa habitualmente, según una escala de Likert de 5 puntos, que oscila entre 1 (falso), 2 (probablemente falso), 3 (ni cierto ni falso), 4 (probablemente verdadero) y 5 (verdadero).

Este inventario mide 7 dimensiones de personalidad, compuestas por 4 de temperamento (evitación del daño, búsqueda de sensaciones nuevas, dependencia a la recompensa, y persistencia) y 3 de carácter (autodirectivo, cooperativo, autotrascendente). La combinación de las dimensiones de temperamento y carácter da lugar a 8 configuraciones de personalidad (melancólico, desorganizado, dependiente, autocrático, creativo, organizado, fanático y ciclotímico/ emotivo).

Se obtuvieron puntuaciones significativamente elevadas en Búsqueda de novedad y significativamente bajas en Persistencia y Autodirectividad, en relación con las puntuaciones tipificadas en la población española.

SCL-90-R (Cuestionario de 90 sintomas). (Symptom Check List90; Revised, Derogatis, 1994).

A partir de un listado de 90 síntomas, el sujeto responde según el grado subjetivo de malestar considerando una escala de valoración con las siguientes opciones de respuesta: 0 (nada), 1 (un poco), 2 (moderadamente), 3 (bastante) y 4 (extremadamente). Proporciona 
información a partir de 3 índices globales de trastorno y 9 dimensiones de sintomatología primaria (somatización, obsesivo-compulsivo, sensibilidad interpersonal, depresión, ansiedad, hostilidad, ansiedad fóbica, ideación paranoide y psicoticismo), ofreciendo una valoración de nivel de psicopatología actual que presenta el sujeto.

Todas las escalas mostraron puntuaciones significativamente elevadas, a excepción de la escala Ansiedad Fóbica.

BDI (Inventario de depresión de Beck) (Beck Depression Inventory, Beck y cols., 1961).

Consta de 21 ítems que el sujeto debe valorar y escoger en una escala de 0 a 3 en función de la gravedad que representa. Evalúa la autopercepción del sujeto frente a diversos aspectos conductuales y cognitivos, proporcionando una medida clínica de la depresión.

La puntuación obtenida por la paciente fue de 33, lo que indicaría un grado de depresión severa según la baremación de esta escala.

\section{ESTABLECIMIENTO DE LAS METAS DEL TRATAMIENTO}

Considerando la información recogida en las entrevistas de evaluación, planteamos junto con la paciente las siguientes metas del tratamiento:

a) Eliminación de los episodios de compra compulsiva.

b) Prevención de recaídas.

c) Mejora de la relación de pareja.

\section{OBJETIVOS TERAPÉUTICOS}

Se establecieron como conductas objeto de intervención los siguientes objetivos terapéuticos:

a) Disminución de los deseos y los pensamientos sobre las conductas de compra compulsiva.

b) Adquisición de habilidades de autocontrol sobre la conducta de compra compulsiva.

c) Identificación de los factores de riesgo que inducen una recaída.

d) Identificación de estrategias adecuadas de actuación ante la aparición de deseos de compra.

e) Desarrollo de nuevos hábitos de consumo. 
f) Realización de actividades alternativas, incompatibles con la conducta de compra compulsiva.

g) Entrenamiento en habilidades de comunicación.

h) Entrenamiento en asertividad.

i) Reestructuración cognitiva de los pensamientos distorsionados.

\section{SELECCIÓN DEL TRATAMIENTO}

\section{Justificaclón teórlca del plan de Intervenclón}

Consideramos que la compra compulsiva se asemeja en muchos aspectos a las drogodependencias 0 al juego patológico. Algunas de las razones que nos llevan a esta conclusión son las siguientes: 1) este trastorno se caracteriza por la presencia de preocupación constante por comprar o por formas de obtener dinero para poder comprar, siendo estos pensamientos egosintónicos; 2) aparece el fenómeno de la progresión, es decir, los episodios de compra ocurren con una mayor ocurrencia, frecuencia y duración, fenómeno similar a la tolerancia; 3) en caso de no poder comprar, aparece irritabilidad y malestar subjetivo; 4) la conducta de compra resulta gratificante para el sujeto, al menos, durante la realización de la misma; 4) existe una sensación de pérdida de control, y 5) la conducta persiste a pesar de que interfiere gravemente en la vida cotidiana. Afirmamos, al igual que Marks (1990), que no es necesaria la presencia de dependencia fisiológica para establecer el diagnóstico de dependencia, puesto que existen rasgos comunes que comparten las adicciones quimicas y las conductuales. Por todo ello, conceptualizamos la compra compulsiva como una adicción psicológica no ligada al uso de sustancias.

El enfoque terapéutico propuesto para la compra compulsiva, según Echeburúa (1999), consistiría en el uso técnicas de control de estímulos, exposición programada a las situaciones de riesgo, solución de problemas especificos, creación de un nuevo estilo de vida y prevención de recaídas. Adès y Leyoyeux (2003), además, proponen el uso de la reestructuración cognitiva, dado que las personas que presentan compra compulsiva manifiestan cogniciones irracionales centradas en los objetos comprados y en las propias capacidades de compra. 
La selección del tipo de tratamiento en el caso clínico de este estudio se estableció en función del análisis funcional de la conducta problema, asl como del metanálisis de publicaciones sobre tratamientos psicológicos de este trastomo.

\section{APLICACIÓN DEL TRATAMIENTO}

Se aplicó un tratamiento estructurado y de tiempo limitado con un total de 23 sesiones. En el anexo 1 puede observarse el contenido de las sesiones terapéuticas según su duración, frecuencia, asistencia (P: paciente; F: paciente y marido), objetivos, técnicas psicologicas y tareas para casa.

Las cuatro primeras sesiones consistieron en sesiones de evaluación. Durante la primera sesión se efectuó una entrevista clínica orientada a establecer una orientación diagnóstica, registrando los datos de la historia clínica y la anamnesis. La segunda y la tercera sesión consistieron en realizar una evaluación psicológica en la que se administraron los tests psicológicos anteriomente citados. Posteriormente, en la cuarta sesión se realizó una devolución de los resultados obtenidos en la exploración psicológica realizada y, considerando todos los datos recogidos, se determinó el tipo de tratamiento más adecuado, explicando a la paciente los objetivos del mismo.

El tratamiento consistió en una terapia de orientación cognitivoconductual, estructurada en 19 sesiones. Debido a que la relación conyugal se hallaba extremadamente deteriorada, decidimos abordar este problema en las tres primeras sesiones de tratamiento como objetivo principal, favoreciendo la adquisición de conductas interpersonales adaptativas. Estas sesiones se realizaron con una frecuencia semanal, asistian ambos miembros de la pareja y la técnica utilizada fue terapia de pareja. El marido se sentía muy irritable y consideraba la posibilldad de incapacitar a $\mathrm{M}$. temporalmente a nivel judicial, de denunciarla o de separarse. Estaba muy preocupado por el hecho de tener que asumir el pago de las deudas de su esposa, ya que esto interfería con la planificación económica que él se habla estructurado con el fin de afrontar su futura jubilación. Comentaba que su trabajo comenzaba a verse afectado, dado que le costaba concentrarse durante el mismo, tenfa dificultades en conciliar el sueño 
y se sentía triste. Sus pautas de comunicación y de interacción conyugal se caracterizaban por usar un estilo agresivo. M. se mostraba muy culpable por lo sucedido, principalmente porque no podía asumir el pago de las deudas económicas contraídas y su familia se veía forzada a solventar estas deudas. La paciente, por un lado, aceptaba cualquier decisión drástica que adoptase su marido y, por otro lado, expresaba sentir miedo a que él se mostrase agresivo hacia ella, tal y como había sucedido en tres ocasiones a lo largo de su matrimonio en las que $M$. había sido agredida físicamente por su marido. Su estilo de comunicación se caracterizaba por la baja asertividad. El distanciamiento emocional de ambos era muy notable. Durante las entrevistas se facilitó un ambiente en el que ambos pudiesen ventilar sus emociones negativas, expresándolas abiertamente, considerando las motivaciones del otro cónyuge y reconduciéndolas de manera que entre sesiones continuasen mostrando tendencia a exteriorizar sus sentimientos asertivamente. Se realizó psicoeducación en tomo a identificar estilos de comunicación asertivos frente a los no asertivos y agresivos. Aconsejamos que no adoptasen ninguna decisión importante que se viese influida por emociones negativas, sino que pospusieran este tipo de decisión hasta poder realizar una valoración objetiva de los pros y los contras de cada opción. Realizamos paralelamente sesiones psicoeducativas sobre la compra compulsiva, conceptualizándola como una adicción psicológica, explicando los objetivos del tratamiento, las pautas terapéuticas y las tareas para casa. Durante estas sesiones se indicó como pauta terapéutica el control de estímulos, es decir, control de gastos (diario y con recibos) y evitación de situaciones y circuitos de riesgo. Provisionalmente, y hasta adquirir autocontrol sobre la conducta de compra compulsiva, M. no podla tener acceso directo a ninguna fuente de obtención de dinero (cartilla del banco, tarjetas de crédito,...). Su marido, que aceptó ejercer de coterapeuta, le debía administrar diariamente una cantidad de dinero prefijada y pactada con $\mathrm{M}$. de antemano, en función de la planificación de los gastos que M. iba a tener durante el día. Del mismo modo, M. debla evitar todas aquellas situaciones de riesgo que le inducian a comprar (calles y tiendas donde solía realizar los episodios de compra compulsiva). Se indicaron como tareas para casa que la paciente rellenara dos 
autorregistros; por un lado, el autorregistro de control de gastos, consistente en recoger información acerca de la fecha, dinero pactado, gastos realizados, diferencia y firma del coterapeuta y, por otro lado, el autorregistro de conducta de compra, donde se obtenía información en torno a la fecha, lugar, ocurrencia o no de episodio de compra, dinero gastado, tiempo empleado, emoción y pensamientos antes, durante y después del episodio. Al final de cada día, M. debía anotar en el autorregistro de control de gastos los gastos realizados de forma detallada, entregar a su marido los recibos de dichos gastos y mostrarle la diferencia entre el dinero pactado y el dinero gastado. En caso de buen cumplimiento de estas pautas, el coterapeuta firmaba en la casilla correspondiente de dicho autorregistro. Paralelamente, la paciente debía cumplimentar diariamente el autorregistro de conducta de compra. En caso de suceder algún episodio de compra, este autorregistro proporcionaría información valiosa para identificar factores desencadenantes $y$, en caso de no comprar, proporcionaría un valor autorreforzante para la paciente a corto plazo.

Las siguientes 16 sesiones se centraron en consolidar las pautas de comunicación interpersonal adecuadas adquiridas $y$, paralelamente, el objetivo principal del abordaje terapéutico fue el mantenimiento de la desaparición de la conducta de compra compulsiva y la prevención de recaídas. Las sesiones se establecieron con una frecuencia semanal, a excepción de las últimas 7 sesiones, que fueron quincenales. La asistencia a estas sesiones consistió en alternar 2 sesiones a las que la paciente acudía sola y 1 sesión a la que acudía acompañada por su marido. Durante estas sesiones realizamos reestructuración cognitiva de aquellas cogniciones desadaptativas que impedían una comunicación fluida entre los miembros de la pareja. Ambos mostraban una marcada tendencia a presentar frecuentemente la inferencia arbitraria como distorsión cognitiva, es decir, sin tener evidencias y a partir de observar el comportamiento de la pareja, realizaban conclusiones erróneas, atribuyendo pensamientos y sentimientos al cónyuge. Se les animó por un lado, a exteriorizar sus propios pensamientos y sentimientos, y por otro lado, a preguntar abiertamente sobre los pensamientos y sentimientos de la pareja, absteniéndose de la tendencia de interpretar las cogniciones, motivaciones y sentimientos del otro. 
Las pautas de control de estímulos se fueron atenuando progresivamente. Indicamos continuar con pautas de control de gastos diario y con justíficantes hasta la sesión 6 (mes y medio). A partir de la sesión 7 y hasta la sesión 10, se indico control de gastos cada 2 días, con recibos (1 mes). Posteriormente, en la sesión 11 y hasta la sesión 13 , se indicó control de gastos semanal con recibos (1 mes). Después, a partir de la sesión 14 y hasta la sesión 16, control de gastos semanal sin recibos (1 mes y medio) y, finalmente, a partir de la sesión 17 y hasta la sesión 19, sin pautas de control de gastos (mes y medio), es decir, se eliminó la tarea para casa de anotar los autorregistros de control de gastos y conducta de compra y se indicó que $M$. fuese asumiendo progresivamente la gestión del dinero sin un control extemo. Cabe señalar que la pauta de control de gastos no supuso para la paciente ni para su marido ninguna dificultad puesto que, como hemos comentado anteriormente, desde años ya tenían costumbre de realizar una contabilidad exhaustiva de los gastos familiares mensuales. En cuanto a la pauta de evitación de situaciones y circuitos peligrosos indicada durante las primeras sesiones de tratamiento, fue $M$. quien informó al principio querer no estar en contacto con todas aquellas situaciones relacionadas con su conducta de compra compulsiva y es por ello que aceptó bien el hecho de evitar circuitos peligrosos. En la sesión 9 informó que en una ocasión había ido por las calles donde estaban las tiendas en las que realizaba compras compulsivas y comentó que estuvo mirando escaparates y que se sintió bien por no haber presentado ganas de comprar. Refirió intranquilidad subjetiva en tomo a si sería capaz de permanecer dentro de una tienda sin presentar deseos por comprar compulsivamente en un futuro.

Diseñamos, junto con la paciente, un plan de exposición en vivo con prevención de respuesta con el fin de incrementar sus expectativas de autoeficacia sobre la conducta de compra compulsiva. Este plan de exposición en vivo consistió en programar, a partir de la sesión 11, exposiciones en vivo de una duración de hora y media y con una frecuencia semanal como mínimo de 2 veces a la semana. M. debía ir sola y sin dinero a tiendas donde solía realizar compras compulsivas en el pasado. Posteriormente, en la sesión 13, se indicó que fuese sola y con el dinero que disponía para pasar un día habitual. Debía anotar en un autorregistro (el autorregistro de exposiciones), la fecha, el lugar 
y tiempo de exposición, nivel de ansiedad antes, durante y después de la exposición y los pensamientos antes, durante y después de la misma. M. mostró una buena adhesión a esta pauta terapéutica, realizando exposiciones cada 3-4 días. Se sentía contenta por no haber presentado ganas de comprar nada. A pesar de que al principio de las exposiciones presentaba cierto grado de ansiedad neurovegetativa, este nivel de ansiedad fue disminuyendo y $\mathrm{M}$. llegó a informar que incluso cogía objetos y se los probaba sin presentar ansiedad.

Alentamos a la paciente a asumir personalmente el pago de las deudas contraídas con el objetivo de fomentar su autorresponsabilidad. Decidió solicitar un crédito a una entidad bancaria con el que, en tres años y medio, solventarla los tres préstamos anteriores y con el que, además, devolvería a su marido el dinero que él había asumido hasta el momento.

En cuanto a la prevención de recaídas, identificamos, junto con M., aquellos estímulos que constitufan posibles factores de riesgo que desencadenaran una recaída, como llevar mucho dinero encima, estar en una tienda donde solía realizar los episodios de compra, discutir con su marido, querer hacer un buen regalo o presentar ánimo disfórico. Asimismo se analizaron las posibles altemativas de afrontamiento adecuadas en caso de presentar en un futuro ganas de comprar compulsivamente. Por ejemplo, decírselo a su hija o a su marido en el mismo momento en que presentara deseos de compra, irse de la tienda, hacer una planificación de qué es lo que quería comprar (según artículo necesitados y según presupuesto aproximado prefijado), escribirto en una lista y ceñirse a esta planificación, no llevar grandes cantidades de dinero encima, realizar actividades alternativas gratificantes que permitieran, por un lado, hacer incompatible la realización de estas actividades con la ejecución de episodios de compra compulsiva y, por otro lado, la satisfacción personal por realizar dichas actividades, con la consiguiente mejora en el estado de ánimo y, por último, fomentar la comunicación adaptativa con su marido.

\section{EVALUACIÓN DE LA EFICACIA DEL TRATAMIENTO}

Desde el inicio del tratamiento, la paciente no presentó ningún episodio de compra compulsiva. Únicamente en la sesión 7 informó 
que habla tenido en una ocasión pensamientos y ganas de comprar, asi como ansias de tener dinero y de salir de casa, desencadenados porque percibia que su marido mostraba una actitud extremadamente rígida y esto le hacla sentirse triste. Su estado de ánimo mejoró notablemente a lo largo de las sesiones: dormia mejor, se sentia más tranquila y, en la sesión 11, refinio que hacla años que no se sentía así. En cuanto inició las sesiones de tratamiento decidió contar su problema a sus hermanas y sobrinos, percibiendo apoyo emocional, aunque comentaba que se vela incapaz de contárselo a sus cuñadas, con quienes mantenía una excelente relación dado que le ofrecieron un apoyo emocional importante tras haber sido mastectomizada años atrás. Más tarde, en la sesión 17, informó que ya había contado a sus cuñadas su problema con las compras y que se sentla mejor, más tranquila, porque todas las personas importantes para ella ya conoclan la existencia de su problema.

En la sesión 12 informó que, su sobrino, hijo de su hermana mayor, había declarado a su mujer que habla contraído una deuda económica elevada por presentar una tendencia a comprar frecuentemente diversos objetos, especialmente artículos de informática y herramientas y que se sentía desbordado emocionalmente. La paciente se implicó en darle pautas de control de estímulos y le animó a que solicitara ayuda profesional. Posteriormente, la paciente nos informó que su sobrino habla sido diagnosticado de compra compulsiva y que también habia iniciado tratamiento psicológico para abordar este problema.

Se realizó una evaluación post-tratamiento al finalizar las sesiones terapéuticas y al cabo de un mes y tres meses, administrando el SCL$90-R$ y el BDI. En las figuras 1 y 2 pueden observarse la evolución en cuanto a la psicopatologla presentada por la paciente tanto en la evaluación pre-tratamiento, como en las evaluaciones post-tratamiento y de seguimiento al mes y tres meses.

Observando los resultados obtenidos en el SCL-90-R (véase figura 1), al comparar las puntuaciones entre el pre-tratamiento y el posttratamiento se observa una mejoría significativa en todas las escalas, especialmente en las escalas Ansiedad, Hostilidad, Ansiedad Fóbica, Obsesivo-Compulsivo, y Sensibilidad Interpersonal. Al mes de seguimiento se observó un perfil ligeramente más elevado en las escalas Ansiedad Fóbica, Hostilidad, Obsesivo-Compulsivo, 


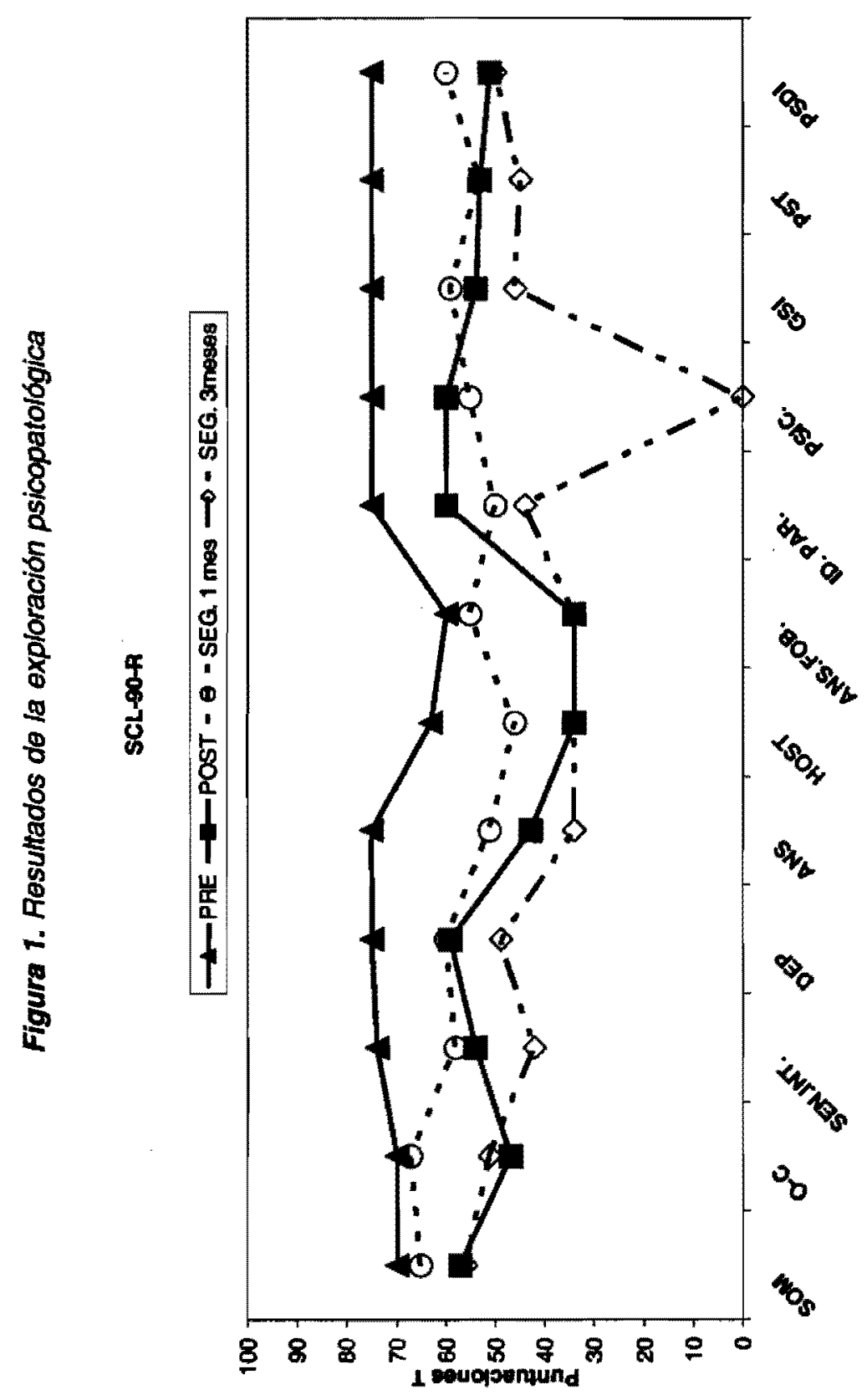


Somatización y Ansiedad, sin ser significativas estas puntuaciones a nivel psicopatológico. La paciente refirió sentir diversas molestias somáticas secundarias al tratamiento con quimioterapia y radioterapia recibido años atrás. Se sentía especialmente muy cansada físicamente y afirmó presentar cogniciones pesimistas centradas en las consecuencias ocasionadas tras haber padecido una neoplasia mamaria. Este hecho podría explicar estos resultados. Por último, considerando las puntuaciones correspondientes a los tres meses de seguimiento, se observa una mejoría global notable en el perfil de las puntuaciones de todas las escalas. Comparando las los resultados obtenidos entre el pre-tratamiento y el seguimiento a los tres meses y analizando las diferentes escalas se observa, especialmente, una disminución en las puntuaciones de las escalas Psicoticismo, Ansiedad, Sensibilidad Interpersonal, Ideación Paranoide.

En la figura 2, que muestra los resultados obtenidos en el BDI, puede observarse una mejoría significativa en el estado anímico tanto al finalizar el tratamiento como en el seguimiento al mes (Pre-tratamiento, PD:33; post-tratamiento, PD: 14; seguimiento al mes, PD: 11; seguimiento a los tres meses, PD: 15).

Figura 2. Resultados del Inventario de Depresión de Beck

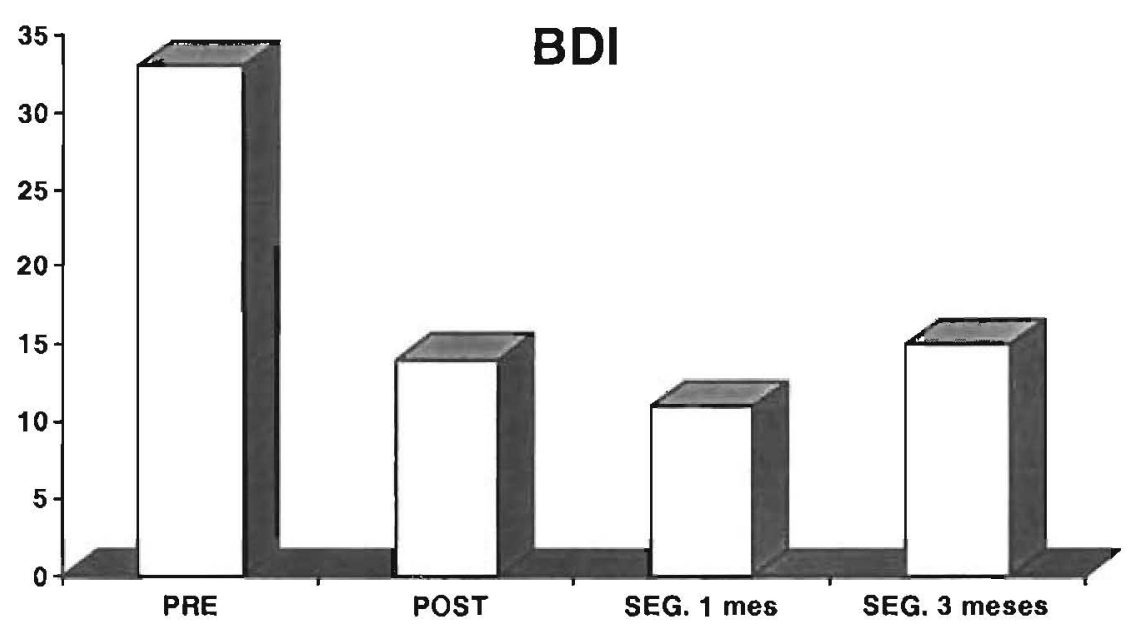


Realizando un análisis cualitativo de los ítems se observa que la paciente, según los resultados obtenidos en la evaluación pre-tratamiento, presentaba cogniciones marcadamente pesimistas en torno al futuro, asi como intensos sentimientos de fracaso personal, insatisfacción, culpa, desprecio a sí misma y autoacusaciones. Asimismo mostraba tendencia al retraimiento social $e$ indecisión al tomar decisiones. Los resultados de las evaluaciones realizadas en el posttratamiento, seguimiento al mes y a los tres meses indican una mejoría notable en cuanto al pesimismo ante el futuro e interés hacia las relaciones sociales. Sin embargo, los sentimientos de fracaso culpa e insatisfacción han mostrado una evolución más lenta, observándose, en el seguimiento a los tres meses, leves sentimientos de fracaso e insatisfacción con su aspecto físico. En la entrevista de seguimiento a los tres meses la paciente refirió sentirse levemente culpable al pensar en el pasado, asl como continuar con sentimientos de insatisfacción con su imagen corporal y preocupada por síntomas somáticos secundarios a la neoplasia mamaria padecida. No obstante se mostraba eutímica, satisfecha y con proyectos de futuro.

En la figura 3, que muestra el nivel de ansiedad experimentado durante las tareas de exposición en vivo con prevención de respuesta, puede apreciarse la evolución que mostró la paciente. Las dos primeras semanas realizo en total 7 exposiciones, sin dinero, presentando una disminución notable del nivel de ansiedad percibido durante las mismas. A partir de la sesión de exposición número 8 , en los que la paciente debla ir con el dinero que disponla para pasar un dia y coincidiendo con control semanal de gastos, estos cambios se mantuvieron. Tras la última sesión de exposición pautada, se indicó a la paciente que continuase con tareas de exposición en vivo, sin rellenar autorregistros, refiriendo en las visitas no mostrar ansiedad ni gánas de comprar. Destacamos la elevada motivación mostrada por la paciente para la realización de esta pauta terapéutica, realizando las exposiciones con intervalos muy cortos, incluso llegando a una frecuencia de cuatro exposiciones por semana.

Al finalizar el tratamiento, $M$. se habla mantenido 10 meses abstinente de la conducta de compra. No habla presentado pensamientos ni deseos por comprar, mostraba unos hábitos de consumo adaptativos y habilidades de autocontrol. Se mostraba eutímica.y sus 
Figura 3. Exposición con prevención de respuesta EXPOSICIÓN EN VIVO

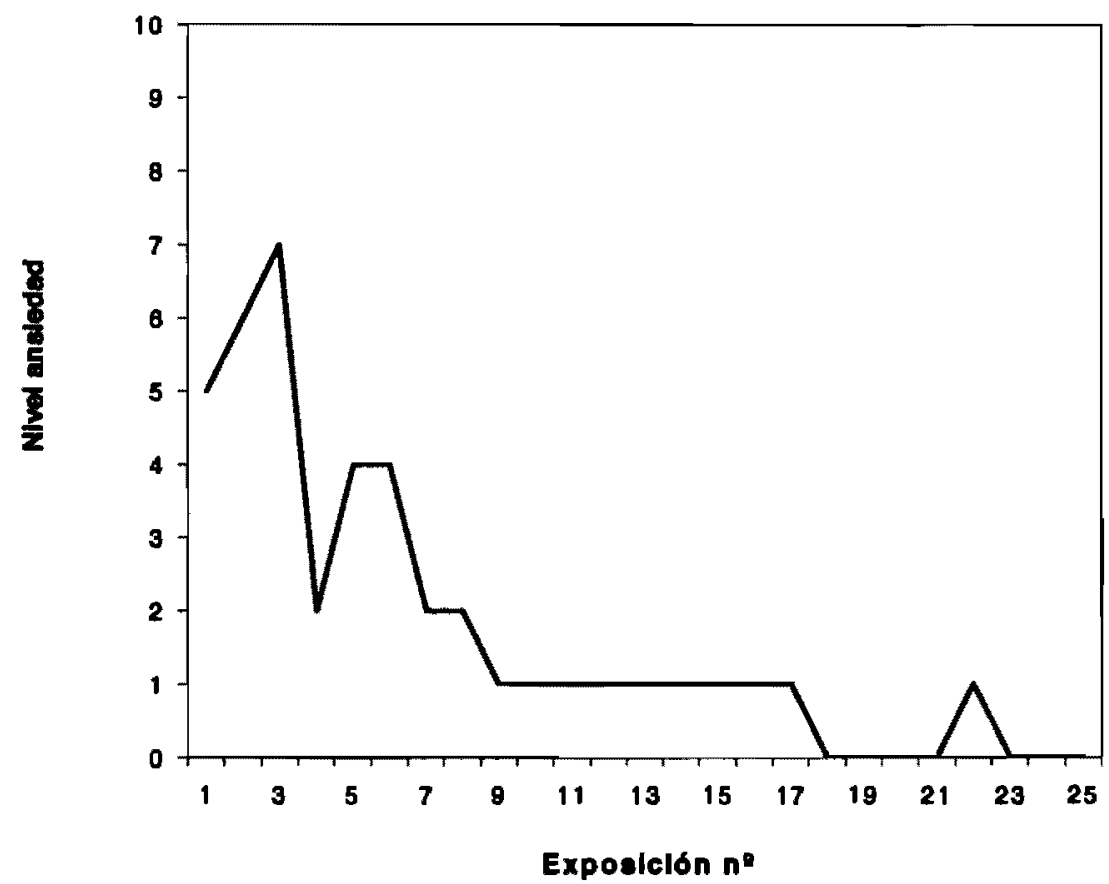

expectativas de autoeficacia fueron incrementándose progresivamente. Refería sentirse muy contenta por poder permanecer en tiendas donde solía realizar los episodios de compra compulsiva sin experimentar ansiedad. Al mes y a los tres meses de seguimiento estos cambios se mantenían.

Cabe señalar que la paciente mostró una adecuada adhesión a las pautas terapéuticas indicadas asl como conciencia de trastómo desde el inicio del tratamiento. Se sentía cómoda realizando las tareas para casa, aceptaba un control extemo de gastos y estaba muy motivada a solucionar sus problemas de compra compulsiva.

La evolución de la relación de pareja fue positiva. Al principio de las sesiones la paciente mostraba una actitud reservada y poco asertiva hacia su marido. Su sentimiento de culpabilidad por lo sucedido generaba que la paciente no se atreviese a pedirle dinero o 
que, ante las demandas exigentes de su marido acerca de conocer todos los detalles de su problema con las compras (exactamente insistía mucho en conocer en qué se había gastado todo ese dinero), ella prefiriese no contarle nada provisionaimente. Expresó que tenía miedo de ser agredida físicamente, puesto que percibía mucha hostilidad hacia ella. Ambos cónyuges adquirieron a lo largo de las sesiones estilos de comunicación interpersonal adaptativos que luego generalizaron en su vida cotidiana. La calidad de la relación matrimonial mejoró notablemente y así lo percibieron ambos cónyuges.

\section{DISCUSIÓN}

El presente estudio, pese a sus limitaciones de generalización por ser un estudio de caso, sugiere que la terapia cognitivo-conductual es efectiva para abordar la compra compulsiva, tal y como apuntan algunos autores (Adès y Lejoyeux, 2003; Echeburúa, 1999).

La aplicación de la técnica de control de estímulos, en el caso clínico que presentamos, pretendio eliminar los episodios de compra a corto plazo y mantener la abstinencia a largo plazo. Se trataba de interrumpir el patrón conductual secuencial que la paciente presentaba ante los estímulos elicitadores de su conducta (disponibilidad de dinero y permanecer en tiendas), y que se caracterizaba por: $1^{9}$ ) surgimiento de deseo por comprar, asociado con malestar subjetivo, $\left.2^{9}\right)$ aparición de un episodio de compra compulsiva, en el que se aliviaría este malestar (refuerzo positivo), $3^{2}$ ) presencia de sentimientos de culpa, arrepentimiento y autorreproches tras la compra realizada y $4^{\circ}$ ), disminución de estas emociones negativas mediante un nuevo episodio de compra (refuerzo negativo). Tanto el control de gastos como la evitación de situaciones de riesgo permitieron, en primer lugar, controlar aquellos estímulos que determinaban que $\mathrm{M}$. presentase episodios de compra compulsiva, reduciendo la probabilidad de ejecución de dicha conducta. En segundo lugar, el control estimular permitio interrumpir el patrón de reforzamiento que mantenía la conducta de compra compulsiva, perdiendo, de este modo, su valor reforzante. Indirectamente, y a lo largo del transcurso de las sesiones, $M$. informó que mediante el control de gastos habia adquirido una capacidad para otorgar mayor valor al dinero. 
Mediante la técnica de prevención de recaldas se proporcionaron estrategias de actuación con las que la paciente pudiese anticipar y/o prevenir nuevos episodios de compra asl como pautas adecuadas de afrontamiento ante la ocurtencia de una recaida. Se trataría de fomentar estrategias de afrontamiento adecuadas, tanto ante desencadenantes extemos como ante desencadenantes intemos, como las emociones negativas, tal y como Miltenberger y cols. (2003) sugieren.

En el presente caso decidimos aplicar la técnica de exposición con prevención de respuesta desde una perspectiva cognitiva, con el fin de incrementar las expectativas de autoeficacia de la paciente. Consideramos, una vez lograda la estabilidad psicopatológica de la paciente y la adecuada evolución mostrada a lo largo de las sesiones, implementar esta técnica mediante exposiciones en vivo, sin presencia de coterapeuta y con una intensidad alta de los estímulos. Rook (1987) considera que el impulso de compra aparece en 1/3 de la población general, y es facilitado e incitado por los anuncios comerciales, el uso de tajetas de crédito, la aparición de empresas que permiten realizar las compras desde casa, la proliferación de los cajeros automáticos y la posibilidad de obtener créditos de forma instantánea. Además, los medios de comunicación ofrecen la fantasía de cómo ha de ser vivida la vida, incitando a los consumidores a «tenerlo todom. Según este autor, en nuestra sociedad de consumo los irnpulsos de compra son nomales y aunque muchas personas, ocasionalmente, compran de una manera no planificada objetos innecesarios, considera que esta conducta no constituiría una patología. Se tratarla de una conducta poco frecuente y que no excederla las propias posibilidades económicas. No obstante, este autor sugiere que la necesidad que conduce a comprar artículos de una manera no planificada es lo que diferencia a las personas que presentan compra incontrolada de los compradores que planifican sus compras. La paciente, en relación con lo expuesto por este autor, mientras compraba incontroladamente y de una manera no planificada, era consciente de que no iba a usar los artículos, pero ante la diversidad de marcas, anuncios comerciales u artículos novedosos se veía incapaz de autocontrolarse. Por ello, expresaba intranquilidad subjetiva en tomo a la capacidad de poder permanecer en una tienda sin experimentar deseos de compra. Tras las tareas de exposición con prevención de respuesta la paciente logró incrementar 
sus expectativas de autoeficacia. No obstante, consideramos que en el tratamiento de las adicciones psicológicas el uso de la técnica de exposición debería ser objeto de futuras líneas de investigación que abordasen su eficacia diferencial con otras técnicas psicológicas y que determinasen qué efectos especificos produce, bien sea el de la extinción de una respuesta emocional condicionada ( $y$, en el caso de las adicciones psicológicas, el identificar el estímulo incondicionado plantea hasta la fecha dificultades de justificación teórica) o bien, el cambio cognitivo logrado a través de la realización de esta técnica.

La paciente presentaba, al finalizar el tratamiento, habilidad para realizar compras desde un criterio racional, comparando diversas alternativas antes de realizar una compra. En relación con lo expuesto, Adès y Lejoyeux (2003) han descrito las fases del proceso de compra de la siguiente forma: 1) Fase de alerta (aparición de las ganas de comprar); 2) Fase de recogida y tratamiento de la información (recopilaclón de información sobre las características del objeto, modelo, precio,...); 3) Fase de evaluación de alternativas (comparación de los productos a escoger); 4) Fase de elección (se concreta la decisión de compra: el objeto, el modelo y lugar de compra); 5) Fase de postcompra (aparecen sentimientos de satisfacción o de decepción). Estos autores sugieren que las personas con compra compulsiva presentan una alteración de las etapas de compra. No aparecen las dos primeras etapas sino que a primera vista el objeto provoca urias ganas irreprimibles de gasto y compra. La evaluación de alternativas no se lleva a cabo de una manera racional que valore las características del objeto, sino en términos afectivos, es decir, el cese de emociones negativas (tensión interna, disforia), o la obtención de placer a corto plazo. En la fase de elección, si aparece duda entre diferentes objetos, la persona con compra compulsiva los compra todos. En la fase de postcompra aparecen sentimientos de decepción y culpabilidad. Los resultados obtenidos en el presente estudio sugieren que las técnicas cognitivoconductuales basadas en el control de estímulos, prevención de recaldas y exposición con prevención de respuesta potencian, indirectamente, la capacidad para evaluar la necesidad de un objeto, comparar las distintas opciones disponibles y realizar una compra considerando toda la información recogida. 
El presente caso clínico se caracterizó por la necesidad de abordar los problemas de pareja y de estabilizar el estado psicopatológico de la paciente paralelamente al problema de compra. Asimismo, destacó el hecho de que, la paciente presentaba antecedentes familiares con compra compulsiva. Por un lado, su padre posiblemente presentaba este trastorno, $y$, por otro lado, un sobrino de la paciente había sido diagnósticado de compra compulsiva. En este sentido cabe destacar los resultados obtenidos en el estudio realizado por Black y cols.(1998), que indicaron que un $9.5 \%$ de los familiares de primer grado de las personas con este trastomo presentaban también compra compulsiva.

Concluimos que será necesario realizar estudios controlados con muestras grandes y con diseño de grupo control que permitan, por un lado, corroborar la eficacia de la terapia cognitivo-conductual y, por otro lado, evaluar la eficacia diferencial de las diferentes técnicas psicológicas. Del mismo modo, el abordaje psicológico de la compra compulsiva plantea la necesidad de determinar qué variables de personalidad y psicopatológicas se asocian a este trastorno.

\section{REFERENCIAS BIBLIOGRÁFICAS}

Aboujaoude, E., Gamel, N. y Koran L.M. (2003). A 1-year naturalistic follow-up of patients with compulsive shopping disorder. Journal of Clinical Psychiatry, 64 (8), 946-950.

American Psychiatric Association: Diagnostic and Statistical Manual of Mental Disorders, Fourth Edition, Text Revision. Washington, DC, American Psychiatric Association, 2000.

Adès,J. y Lejoyeux, M. (2003). La fiebre de las compras. En J. Adès y $M$. Lejoyeux (eds.). Las nuevas adicciones. Internet, sexo, juego, deporte, compras, trabajo, dinero. Barcelona: Kairós. (pp. 21 62). (Orig. 2001).

Beck, A.F., Ward, C.H., Mendelson, M., Mock, J. y Erbaugh, J. (1961). An inventory for measuring depression. Archaics of General Psychopathology, 4: 561-571.

Black, D.W. (1996). Compulsive buying: a review. Joumal of Clinical Psychiatry, 57, (8), 50-55.

Black, D.W., Monahan, P.y Gabel, J. (1997). Fuvoxamine in the treatment of compulsive buying. Joumal of Clinical Psychiatry, 58, 159-163. 
Black, D.W., Repertinger, S., Gaffney, GR. y Gabel, J. (1998). Family History and Psychiatric Comorbidity in Persons with Compulsive Buying: Preliminary Findings. American Joumal of Psychiatry, 155 (7), 960-963.

Black, D.W., Monahan, P., Schlosser, S. y Repertinger, S. (2001). Compulsive buying severity. An analysis of compulsive buying scale results in $\mathbf{4 4}$ subjects. Journal of Nervous and Mental Desease, 189, 123-126.

Bleuler, E. Textbook of Psychiatry. (1924). McMillan. New York.

Christenson, GA., Faber, R.J., de Zwaan, M., Raymond, N., Specker, S., Ekern, M., McKenzie, T., Crosby, R., Crow, S., Eckert, E., Mussell, M. y Mitchell, J. (1994). Compulsive buying: descriptive characteristics and psychiatric comorbidity. Joumal of Clinical Psychiatry, 55, 5-11.

Cloninger, R. (ed.). (1999). Personality and psychopathology. Washington, DC: American Psychiatric Press.

Derogatis, L.R. (1994). Cuestionario de 90 síntomas. TEA Ediciones. Madrid, 2002.

Dittmar, H. y Drury, J. (2000). Self-Image. Is it in the bag?. A qualitative comparison between ordinary and excessive consumers. Joumal of Economic Psychology, 21, 109-142.

Echeburúa, E. (2000). ¿Adicciones... sin drogas?. Las nuevas adicciones: Juego, Sexo, Comida, Compras, Trabajo, Intemet... (2 Ed.). Bilbao: Desclée de Brouwer.

Elliott, R. (1994). Addictive consumption: Function and fragmentation in postmodemity. Joumal of Consumer Policy, 17, 159-179.

Eysenck, S.B.G., Pearson, P.R., Easting, G. y Allsopp, J.P. (1985). Age norms for impulsiveness, venturesomeness and empathy in adults. Personality and Individual Differences, 6: 613-619.

Eysenck, H.J. y Eysenck, S.B.G. (1991). Manual of the Eysenck Personality Scales. Hodder and Stoughton, London.

Faber, R.J., O'Guinn, T.C. y Krych, R. (1987). Compulsive consumption. Advances in Consumer Research, 14, 132-135.

Faber, R.J. y O'Guinn, T.C. (1992). Aclinical screener for compulsive buying. Journal of Consumer Research, 19, 459-569.

Faber, R.J. (1994). Money changes everything: Compulsive buying from a biopsychosocial perspective. American Behavioral Scientist, 35 (6), 809-819. 
Glatt, M.M. y Cook, C.H. (1987). Pathological spending as a form of psychological dependence. British Journal of Addiction, 82: 1257-1258.

Gutiérrez-Zotes, J.A., Bayón, C, Montserrat, C., Valero, J., Labad, A., Cloninger, R.C., Fernández-Aranda, F. (2004). Inventario del Temperamento y el Carácter-Revisado. (TCI-R). Baremación y datos normativos en una muestra de la población general. Actas españolas de psiquiatría, 32(1), 8-15.

Hathaway, S.R. y Mckinley, J.C. (1989). Inventario Multifásico de Personalidad de Minnesota-2. (MMPI-2). TEA Ediciones. Madrid, 2000.

Krych, R. (1989). Abnormal consumer behaviour: a model of addictive behaviours. Advances in consumer research, 16, 745-748.

Krueger.D.W. (1988). On compulsive shopping and spending: a psychodynamic inquiry. American Journal of Psychotherapy; 42(4):574584.

Lawrence, L. (1990). The psychodynamics of the compulsive female shopper. American Journal of Psychoanalysis, 50, 67-70.

Marks I. (1990). Behavioural (non chemical) addictions. British Journal of Addiction, 85, 1389-1394.

McElroy, S.L., Satlin, A., Pope, H., Keck, P., Hudson, J. (1991). Treatment of compulsive shopping with antidepressants: a report of three cases. Annals of Clinical Psychiatry, 3, 199-204.

McElroy S.L., Keck, P.E., Pope, H.G., Smith, J.M. y Strakowski, S.M. (1994). Compulsive buying: a report of 20 cases. Journal of Clinical Psychiatry; 55: 242-248.

Millon, T. (1990). Inventario Clínico Multiaxial de Millon (II). TEA Ediciones. Barcelona, 1996.

Miltenberger, R.G, Redlin, J., Crosby, R., Stickney, M., Mitchell, J., Wonderlich, R.F. y Smyth, J. (2003). Direct and retrospective assessment of factors contributing to compulsive buying. Joumal of Behaviour Therapy and Experimental Psychiatry, 34, 1-9.

Ninan, P., McElroy, S., Kane, C., Knight, B., Casuto, L., Rose, S., Marsteller, F. y Nemeroff, C. (2000). Placebo-controlled study of fluvoxamine in the treatment of patients with compulsive buying. Joumal of Clinical Psychofarmacology, 20, 362-366.

O'Guinn, T.C. y Faber, R.J. (1989). Compulsive buying: a phenomenological exploration. Journal of Consumer Research, 16, 147 157. 
Rook, D.W. (1987). The buying impulse. Journal of Consumer Research, 14,189-199.

Schlosser, S., Black, D.W., Repertinger, S. y Freet, D. (1994). Compulsive buying: demography, phenomenology, and comorbility in 46 subjects. General Hospital Psychiatry, 16, 205-212.

Winestine, M. (1985). Compulsive shopping as a derivative of childhood seduction. The Psychoanalytic Quarterly, 54, 70-72.

ANEXO 1: Estructura de las sesiones

\begin{tabular}{|c|c|c|c|c|}
\hline $\begin{array}{l}\text { Asistencia } \\
\text { por seslon }\end{array}$ & $\begin{array}{l}\text { Frecuencia y } \\
\text { duración }\end{array}$ & Objetivos & Ténicas & $\begin{array}{c}\text { Tareas para } \\
\text { casa }\end{array}$ \\
\hline \multicolumn{5}{|c|}{ EVALUACIÓN } \\
\hline $\begin{array}{ll}1 & \text { P y F } \\
2 & P \\
3 & P \\
4 & P\end{array}$ & $\begin{array}{l}\text { Frecuencia: } \\
\text { lvez/semana } \\
\text { Duración: } 60 \\
\text { min. }\end{array}$ & $\begin{array}{l}\text {-Orientación diagnóstica } \\
\text {-Evaluación psicológica I } \\
\text {-Evaluación psicológica II } \\
\text { - Devolución resultados y } \\
\text { elección del tratamiento }\end{array}$ & $\begin{array}{l}\text {-Entrevista clinica } \\
\text {-Tests psicometricos }\end{array}$ & $=$ \\
\hline \multicolumn{5}{|c|}{ TRATAMIENTO } \\
\hline $\begin{array}{ll}\text { PyF } & \text { Py } \\
2 & \text { PyF } \\
3 & \text { PyF }\end{array}$ & $\begin{array}{l}\text { Frecuencia: } \\
\text { Ivez/semana } \\
\text { Duración: } 40 \\
\text { min. }\end{array}$ & $\begin{array}{l}\text {-Mejora pautas interacción } \\
\text { de pareja } \\
\text {-Eliminación episodios } \\
\text { compra compulsiva }\end{array}$ & $\begin{array}{l}\text {-Terapia de pareja } \\
\text {-Psicoeducación } \\
\text {-Entr. asertividad } \\
\text {-Entr. habilidades } \\
\text { comunicación } \\
\text {-Control estímulos }\end{array}$ & $\begin{array}{l}\text {-Autorregistros: } \\
\text { conducta de } \\
\text { compra } \\
\text { control de } \\
\text { gastos }\end{array}$ \\
\hline $\begin{array}{ll}4 & \mathrm{P} \\
5 & \mathrm{P} \\
6 & \mathrm{P} \mathrm{y} \mathrm{F} \\
7 & \mathrm{P} \\
8 & \mathrm{P} \\
9 & \mathrm{P} \mathrm{y} \mathrm{F} \\
10 & \mathrm{P} \\
11 & \mathrm{P}\end{array}$ & $\begin{array}{l}\text { Frecuencia: } \\
\text { semanal } \\
\text { Duración: } 40 \\
\text { min. }\end{array}$ & \multirow{2}{*}{$\begin{array}{l}\text {-Consolidación pautas } \\
\text { comunicación adaptativas } \\
\text {-Eliminación episodios } \\
\text { compra compulsiva } \\
\text {-Disminución } \\
\text { pensamientos y ganas por } \\
\text { comprar } \\
\text {-Asumir deudas } \\
\text {-Adquisición habilidades } \\
\text { autocontrol } \\
\text {-Reestructuración } \\
\text { cognitiva } \\
\text {-Prevención recardas } \\
\text {-Nuevos hábitos de } \\
\text { compra }\end{array}$} & \multirow[t]{2}{*}{$\begin{array}{l}\text {-Terapia de pareja } \\
\text {-Entr. asertividad } \\
\text {-Control estimulos } \\
\text {-Exposición en vivo } \\
\text {-Situaciones de riesgo } \\
\text { y su afrontamiento } \\
\text {-Actividades } \\
\text { alternativas }\end{array}$} & $\begin{array}{l}\text {-Autorregistros: } \\
\text { conducta de } \\
\text { compra } \\
\text { control de } \\
\text { gastos } \\
\text { exposiciones } \\
\text {-Prética } \\
\text { asertividad }\end{array}$ \\
\hline $\begin{array}{l}13 \mathrm{P} \\
14 \mathrm{P} \\
15 \mathrm{P} \text { y F } \\
16 \mathrm{P}\end{array}$ & $\begin{array}{l}\text { Frecuencia: } \\
\text { Quincenal } \\
\text { Duración: } 40 \\
\text { min. }\end{array}$ & & & \\
\hline $\begin{array}{l}17 \mathrm{P} \\
18 \mathrm{P} \\
19 \mathrm{PyF}\end{array}$ & $\begin{array}{l}\text { Frecuencia: } \\
\text { quincenal } \\
\text { Duración: } 40 \\
\text { min. }\end{array}$ & $\begin{array}{l}\text {-Autocontrol conducta } \\
\text { compra } \\
\text {-Control interno gastos } \\
\text { - Nuevos hábitos de } \\
\text { compra }\end{array}$ & $\begin{array}{l}\text {-No control estímulos } \\
\text {-Exposición en vivo }\end{array}$ & $\begin{array}{l}\text {-Autorregistro: } \\
\text { exposiciones }\end{array}$ \\
\hline
\end{tabular}

P: paciente sola.

$P$ y F: paciente y manido. 